\title{
Divergence-Free Wavelet Frames
}

\author{
Emrah Bostan, Student Member, IEEE, Michael Unser, Fellow, IEEE, and John Paul Ward
}

\begin{abstract}
We propose an efficient construction of wavelet frames in any number of dimensions that are divergence-free. Our approach is based on applying the Leray projector, which is scale-invariant, to a standard wavelet frame. We prove that the projected wavelets retain the basic characteristics (decay rate and order of vanishing moments) of the initial wavelets. Since the Leray projector is also shift-invariant, it is defined as a Fourier multiplier, and our construction is implemented efficiently using the fast Fourier transform. In order to illustrate the practicality of the method, we present vector field denoising experiments.
\end{abstract}

Index Terms-Denoising, divergence-free wavelets, flow MRI, phase-contrast MRI, soft-thresholding, tight wavelet frames, vector fields, vector wavelets, wavelet denoising.

\section{INTRODUCTION}

$\mathbf{T}$ HE mass conservation principle implies that the velocity fields representing incompressible fluid flows have zero divergence. Therefore, these physical systems are often represented using divergence-free wavelets. Not only are they useful for the multi-scale analysis of such phenomena, but also fundamentally prominent for studying the incompressible solutions of the Navier-Stokes equations [1]. Accordingly, they are used for the numerical simulation of incompressible flows [2]. This type of wavelet is relevant for imaging modalities such as phasecontrast magnetic resonance imaging (PCMRI). PCMRI provides three-dimensional and time-resolved blood flow measurements [3]. Since blood flow is incompressible, noise-like errors are reduced by identifying (and eventually suppressing) non-divergence-free components [4], [5]. Volumetric particle image velocimetry (vPIV) techniques are also capable of measuring three-dimensional velocity fields. Similar ideas have recently been applied to vPIV data by removing spurious divergence through a redundant atomic signal decomposition [6].

The design of divergence-free wavelets was considered first by Battle and Federbush [7] and Lemarié-Rieusset [8]. The development in [7] generated orthogonal wavelets (in the 2and 3-dimensional cases) with exponential decay. On the other

Manuscript received December 08, 2014; accepted December 27, 2014. Date of publication January 06, 2015; date of current version January 15, 2015. E. Bostan and J. P. Ward contributed equally to this work. This work was supported in part by the Center for Biomedical Imaging of the Geneva-Lausanne Universities and EPFL. The research leading to these results has received funding from the European Research Council under the European Union's Seventh Framework Programme FP7/2007-2013/ERC grant agreement 267439. The associate editor coordinating the review of this manuscript and approving it for publication was Prof. Alexandre X. Falcao.

The authors are with the Biomedical Imaging Group, École polytechnique fédérale de Lausanne (EPFL), CH-1015 Lausanne VD, Switzerland (e-mail: emrah.bostan@epfl.ch; john.ward@epfl.ch; michael.unser@epfl.ch).

Color versions of one or more of the figures in this paper are available online at http://ieeexplore.ieee.org.

Digital Object Identifier 10.1109/LSP.2015.2388794 hand, the construction in [8] resulted in compactly-supported biorthogonal wavelets. Their construction is based on two pairs of wavelets and scaling functions satisfying certain integration and differentiation relations. An efficient (tensor-product based) implementation of these wavelets was developed by Deriaz and Perrier [9]. Stevenson [10] and Kadri-Harouna and Perrier [11] have extended these constructions to domains with free-slip boundary conditions. Other basis constructions have also been considered [12].

All of the previous schemes are rather technical as they are concerned with divergence-free wavelet basis functions. In this letter, we loosen this constraint by allowing redundancy. Based on this relaxation, we propose a simple yet elegant approach for constructing divergence-free wavelet frames. Our design involves projecting a wavelet frame onto the space of zero divergence vector-valued functions. We prove that the decay rate and order of vanishing moments of the initial wavelets are inherited by their divergence-free counterparts. We employ our framework for the vector field denoising problem both in the two- and three-dimensional settings. In the remaining sections, we shall concentrate on divergence-free tight wavelet frames since they are advantageous for such applications. However our method applies for constructing divergence-free wavelet frames in a more general sense.

\section{A. Notation}

We denote the space of square integrable vector fields (or vector-valued functions) on $\mathbb{R}^{d}$ by $\left(L_{2}\left(\mathbb{R}^{d}\right)\right)^{d}$. A vector field $\boldsymbol{f} \in\left(L_{2}\left(\mathbb{R}^{d}\right)\right)^{d}$ is represented as $\boldsymbol{f}=\left(f_{1} f_{2} \ldots f_{d}\right)^{\top}$, where each component is a function in $L_{2}\left(\mathbb{R}^{d}\right)$. The inner product is defined as follows:

$$
\begin{aligned}
\langle\boldsymbol{f}, \mathbf{g}\rangle & =\sum_{n=1}^{d}\left\langle f_{n}, g_{n}\right\rangle \\
& =\sum_{n=1}^{d} \int_{\mathbb{R}^{d}} f_{n}(\boldsymbol{x}) g_{n}(\boldsymbol{x}) \mathrm{d} \boldsymbol{x} .
\end{aligned}
$$

The Fourier transform of a vector field is the component-wise Fourier transform

$$
\begin{aligned}
\mathcal{F}\{\boldsymbol{f}\}(\boldsymbol{\omega}) & =\widehat{\boldsymbol{f}}(\boldsymbol{\omega}) \\
& =\left(\widehat{f}_{1}(\boldsymbol{\omega}) \widehat{f}_{2}(\boldsymbol{\omega}) \ldots \widehat{f}_{d}(\boldsymbol{\omega})\right)^{\top}
\end{aligned}
$$

where

$$
\widehat{f}_{n}(\boldsymbol{\omega})=\int_{\mathbb{R}^{d}} f_{n}(\boldsymbol{x}) \mathrm{e}^{-2 \pi \mathrm{j} \boldsymbol{x} \cdot \boldsymbol{\omega}} \mathrm{d} \boldsymbol{x} .
$$


The divergence of $\boldsymbol{f} \in\left(L_{2}\left(\mathbb{R}^{d}\right)\right)^{d}$ is given by

$$
\begin{aligned}
\operatorname{div} \boldsymbol{f} & =\nabla \cdot \boldsymbol{f} \\
& =\sum_{n=1}^{d} \frac{\partial f_{n}}{\partial x_{n}} \\
& =\left(\frac{\partial}{\partial x_{1}} \frac{\partial}{\partial x_{2}} \cdots \frac{\partial}{\partial x_{d}}\right)\left(f_{1} f_{2} \ldots f_{d}\right)^{\top} .
\end{aligned}
$$

We denote the collection of divergence-free, square-integrable vector fields as

$$
\mathcal{H}\left(\mathbb{R}^{d}\right)=\left\{\boldsymbol{f} \in\left(L_{2}\left(\mathbb{R}^{d}\right)\right)^{d}: \operatorname{div} \boldsymbol{f} \in L_{2}\left(\mathbb{R}^{d}\right), \operatorname{div} \boldsymbol{f}=0\right\} .
$$

The Leray projector $\mathcal{P}:\left(L_{2}\left(\mathbb{R}^{d}\right)\right)^{d} \rightarrow\left(L_{2}\left(\mathbb{R}^{d}\right)\right)^{d}$ is the unique orthogonal projector that maps a vector field to its divergencefree version [13]. It is defined in the Fourier domain as

$$
\mathcal{F}\{\mathcal{P} \boldsymbol{f}\}(\boldsymbol{\omega})=(\mathrm{I}-\mathrm{P}(\boldsymbol{\omega})) \widehat{\boldsymbol{f}}(\boldsymbol{\omega})
$$

where $\mathrm{I}$ is the $d \times d$ identity matrix and $\mathrm{P}$ is the $d \times d$ matrix

$$
\mathrm{P}(\boldsymbol{\omega})=|\boldsymbol{\omega}|^{-2}\left(\omega_{1} \omega_{2} \ldots \omega_{N}\right)^{\top}\left(\omega_{1} \omega_{2} \ldots \omega_{N}\right) .
$$

Proposition 1: The Leray projector $\mathcal{P}$ is self-adjoint and is invariant to translation and scaling in the sense that

$$
\mathcal{P}\{\boldsymbol{f}(\cdot / a-\mathbf{b})\}(\mathbf{x})=\mathcal{P}\{\boldsymbol{f}\}(\mathbf{x} / a-\mathbf{b}),
$$

for any $\boldsymbol{f} \in\left(L_{2}\left(\mathbb{R}^{d}\right)\right)^{d}, a \in \mathbb{R}^{+}$, and $\mathbf{b} \in \mathbb{R}^{d}$.

Proposition 1 implies that the Leray projector maps a wavelet to another wavelet. In the sequel, we shall use this for our construction of divergence-free wavelet frames.

\section{WAVELETS}

The construction of a band-limited mother wavelet $\phi$ for a tight wavelet frame

$$
\left\{2^{j d / 2} \phi\left(2^{j} \boldsymbol{x}-\boldsymbol{k}\right): j \in \mathbb{Z}, \boldsymbol{k} \in \mathbb{Z}^{d}\right\}
$$

of $L_{2}\left(\mathbb{R}^{d}\right)$ is straightforward, cf. Theorem 12.2.1 of [14]. Recall that a tight wavelet frame satisfies an energy preservation relationship

$$
\|f\|_{L_{2}\left(\mathbb{R}^{d}\right)}^{2}=\sum_{j \in \mathbb{Z}, \boldsymbol{k} \in \mathbb{Z}^{d}}\left|\left\langle f, 2^{j d / 2} \phi\left(2^{j} \cdot-\boldsymbol{k}\right)\right\rangle\right|^{2},
$$

and it is dual to itself

$$
f(\boldsymbol{x})=\sum_{j \in \mathbb{Z}, \boldsymbol{k} \in \mathbb{Z}^{d}}\left\langle f, 2^{j d / 2} \phi\left(2^{j} \cdot-\boldsymbol{k}\right)\right\rangle 2^{j d / 2} \phi\left(2^{j} \boldsymbol{x}-\boldsymbol{k}\right) .
$$

From a scalar-valued frame, we can easily construct band-limited tight frames of $\left(L_{2}\left(\mathbb{R}^{d}\right)\right)^{d}$. The vector-valued frames are obtained as follows. For $n \in \mathbb{N}_{\leq d}$, let $\boldsymbol{e}_{n}$ denote the $n$th unit vector in $\mathbb{R}^{d}$.

\section{Proposition 2: The Collection}

$$
\left\{\boldsymbol{\psi}_{j, \boldsymbol{k}, n}:=2^{j d / 2} \phi\left(2^{j} \cdot-\boldsymbol{k}\right) \boldsymbol{e}_{n}: j \in \mathbb{Z}, \boldsymbol{k} \in \mathbb{Z}^{d}, n \in \mathbb{N}_{\leq d}\right\}
$$

is a vector-valued tight frame of $\left(L_{2}\left(\mathbb{R}^{d}\right)\right)^{d}$.
The next step is to derive a divergence-free tight frame by applying the Leray projector.

Proposition 3: The Collection

$$
\left\{\mathcal{P} \boldsymbol{\psi}_{j, \boldsymbol{k}, n}: j \in \mathbb{Z}, \boldsymbol{k} \in \mathbb{Z}^{d}, n \in \mathbb{N}_{\leq d}\right\}
$$

is a tight frame of $\mathcal{H}\left(\mathbb{R}^{d}\right)$. In particular, for $\boldsymbol{f} \in \mathcal{H}\left(\mathbb{R}^{d}\right)$

$$
\boldsymbol{f}=\sum_{j \in \mathbb{Z}, \boldsymbol{k} \in \mathbb{Z}^{d}, n \in \mathbb{N}_{\leq d}}\left\langle\boldsymbol{f}, \mathcal{P} \boldsymbol{\psi}_{j, \boldsymbol{k}, n}\right\rangle \mathcal{P} \boldsymbol{\psi}_{j, \boldsymbol{k}, n} .
$$

Proof: Let $\boldsymbol{f} \in \mathcal{H}\left(\mathbb{R}^{d}\right)$. Since the Leray projector is selfadjoint, we have

$$
\begin{aligned}
\|\boldsymbol{f}\|_{\mathcal{H}\left(\mathbb{R}^{d}\right)}^{2} & =\|\boldsymbol{f}\|_{\left(L_{2}\left(\mathbb{R}^{d}\right)\right)^{d}}^{2} \\
& =\|\mathcal{P} \boldsymbol{f}\|_{\left(L_{2}\left(\mathbb{R}^{d}\right)\right)^{d}}^{2} \\
& =\sum_{j \in \mathbb{Z}, \boldsymbol{k} \in \mathbb{Z}^{d}, n \in \mathbb{N}_{\leq d}}\left|\left\langle\mathcal{P} \boldsymbol{f}, \boldsymbol{\psi}_{j, \boldsymbol{k}, n}\right\rangle\right|^{2} \\
& =\sum_{j \in \mathbb{Z}, \boldsymbol{k} \in \mathbb{Z}^{d}, n \in \mathbb{N}_{\leq d}}\left|\left\langle\boldsymbol{f}, \mathcal{P} \boldsymbol{\psi}_{j, \boldsymbol{k}, n}\right\rangle\right|^{2} .
\end{aligned}
$$

\section{A. Decay Estimates}

An important property of our construction is that we preserve the advantageous properties of the initial wavelet $\phi$. Both vanishing moments and decay rates remain unchanged.

Theorem 4: Let $\phi$ be a differentiable function with vanishing moments of order $N \geq 1$ such that $\phi$ and its derivatives satisfy the decay estimates

1) $|\phi(\boldsymbol{x})| \leq C(1+|\boldsymbol{x}|)^{-d-N+\epsilon}$

2) $\left|\mathrm{D}^{\alpha} \phi(\boldsymbol{x})\right| \leq C(1+|\boldsymbol{x}|)^{-d-N-1+\epsilon}, \quad|\alpha|=1$,

for some $C>0$ and $0 \leq \epsilon<1$. Then the components of the divergence-free wavelets $\mathcal{P} \boldsymbol{\psi}_{0,0, n}$ have the same number of vanishing moments and similar decay to $\phi$, i.e., for $n=1, \ldots, d$

$$
\left(\boldsymbol{e}_{n} \cdot \mathcal{P} \boldsymbol{\psi}_{0, \mathbf{0}, n}\right)(\boldsymbol{x}) \leq C(1+|\boldsymbol{x}|)^{-d-N+\epsilon^{\prime}}
$$

for some $0 \leq \epsilon^{\prime}<1$.

Proof: This result follows from the analysis of singular-integral operators that was presented in [15]. As each component is similar, we only consider $n=1$. In the Fourier domain,

$$
\begin{aligned}
\mathcal{F}\left\{\mathcal{P} \boldsymbol{\psi}_{0,0,1}\right\}(\boldsymbol{\omega}) & =(\mathrm{I}-\mathrm{P}(\boldsymbol{\omega})) \boldsymbol{e}_{1} \widehat{\phi}(\boldsymbol{\omega}) \\
& =-\widehat{\phi}(\boldsymbol{\omega})|\boldsymbol{\omega}|^{-2} \sum_{n=1}^{d} \omega_{1} \omega_{n}
\end{aligned}
$$

Since $\left.|| \boldsymbol{\omega}\right|^{-2} \sum_{n=1}^{d} \omega_{1} \omega_{n} \mid \leq 1$, the vanishing moments are preserved. Furthermore, $\sum_{n=1}^{d} \omega_{1} \omega_{n}$ is a homogeneous harmonic polynomial of degree 2 , so we can decompose the multiplier in the spherical harmonics of degree $2\left\{Y_{2, m}\right\}_{m=1}^{d}$ as

$$
|\boldsymbol{\omega}|^{-2} \sum_{n=1}^{d} \omega_{1} \omega_{n}=\sum_{m=1}^{d} c_{m} Y_{2, m}\left(\frac{\boldsymbol{\omega}}{|\boldsymbol{\omega}|}\right)
$$

for some coefficients $c_{m}$. The decay estimates now follow from [16, Theorem 3.2]. 


\section{B. Wavelet Construction Details}

The particular wavelet $\phi$ that we use for implementation is a Meyer-type mother wavelet. It is defined in the Fourier domain by a radial profile function $h$, i.e., $\widehat{\phi}(\boldsymbol{\omega})=h(|\boldsymbol{\omega}|)$, where

$$
h(r)=\left\{\begin{array}{cl}
\cos \left(2 \pi q_{m}(r)\right), & \frac{1}{8}<r \leq \frac{1}{4} \\
\sin \left(2 \pi q_{m}\left(\frac{r}{2}\right)\right), & \frac{1}{4}<r \leq \frac{1}{2} \\
0, & \text { otherwise }
\end{array}\right.
$$

and $q_{m}$ is a degree $2 m+1$ polynomial satisfying the following: $q_{m}(1 / 8)=0, q_{m}(1 / 4)=0$, and $q_{m}^{(j)}(1 / 8)=q_{m}^{(j)}(1 / 4)=0$ for $j=1, \ldots, m$, cf. [17]. In our experiments, we set the parameter $m=2$. Our divergence-free wavelets are constructed as described above. We generate the scalar-valued tight frame associated with $\phi$; extend it to a vector-valued frame; and finally, apply the Leray projector to each frame element. As for the implementation of our frame, we follow the pyramid construction of [18]. Our method is efficient as it has a complexity of $\mathcal{O}\left(\mathrm{N}^{3} \log \mathrm{N}\right)$ with $\mathrm{N}^{3}$ being the number of voxels.

\section{VECTOR FIELD REgUlARIZATION}

We now consider the problem of restoring incompressible fields from noisy measurements. Our goal is to denoise a discrete vector field denoted by $\mathbf{f}$ from the observation

$$
\mathbf{y}=\mathbf{f}+\mathbf{n}
$$

where $\mathbf{n}$ is assumed to be additive white Gaussian noise (AWGN). Using a wavelet tight frame, the unknown field is expressed as $\mathbf{f}=\mathbf{W}^{\top} \mathbf{w}$, where $\mathbf{W}$ expands the field in the divergence-free frame. We obtain the expansion coefficients $\hat{\mathbf{w}}$ of the denoised field via the $\ell_{1}$-regularized least-squares problem

$$
\begin{aligned}
\hat{\mathbf{w}} & =\arg \min _{\mathbf{w}}\left\{\frac{1}{2}\|\mathbf{w}-\mathbf{W} \mathbf{y}\|_{2}^{2}+\lambda\|\mathbf{w}\|_{1}\right\} \\
& =\eta_{\lambda}(\mathbf{W} \mathbf{y})
\end{aligned}
$$

where the soft-thresholding function $\eta_{\lambda}(\cdot)$ (with $\lambda>0$ being the threshold value) is applied componentwise [19]. The denoised vector field is then given by $\hat{\mathbf{f}}=\mathbf{W}^{\top} \hat{\mathbf{w}}$.

\section{RESUlTS}

For our experiments, we generate two different band-limited vector field phantoms that have zero divergence (see Fig. 4(a) and (d)). Our 2-dimensional model (called circle) is of size $128^{2} \times 2$, whereas the volumetric one (called tube\&torus) is of size $64^{3} \times 3$. The noisy measurements are obtained by degrading the data with AWGN to achieve various levels of signal-to-noise ratio (SNR). We measure the denoising performance of a given denoising method in terms of $\triangle \mathrm{SNR}$, which simply denotes the improvement provided. Also, for each wavelet-based method, the soft-thresholding operator is applied only to the wavelet (or detail) coefficients. We note that all visualizations are rendered with ParaView (Kitware Inc.) [20].
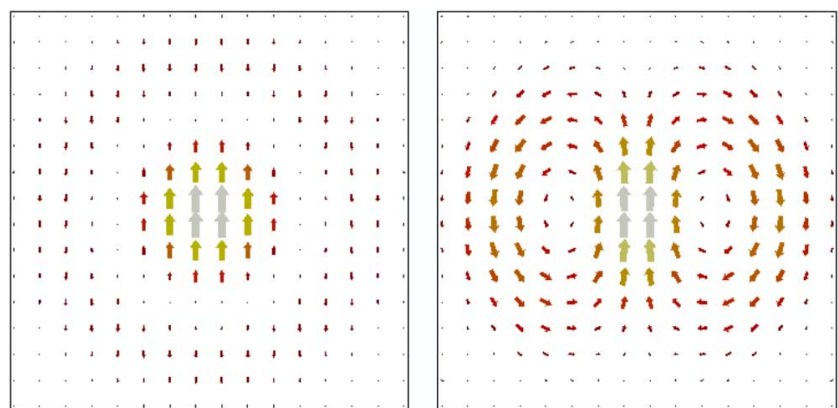

Fig. 1. Vector field representation of $\boldsymbol{\psi}_{1, \mathbf{0}, 1}$ (left) and its Leray projection $\mathcal{P} \boldsymbol{\psi}_{1, \mathbf{0}, 1}$ (right)

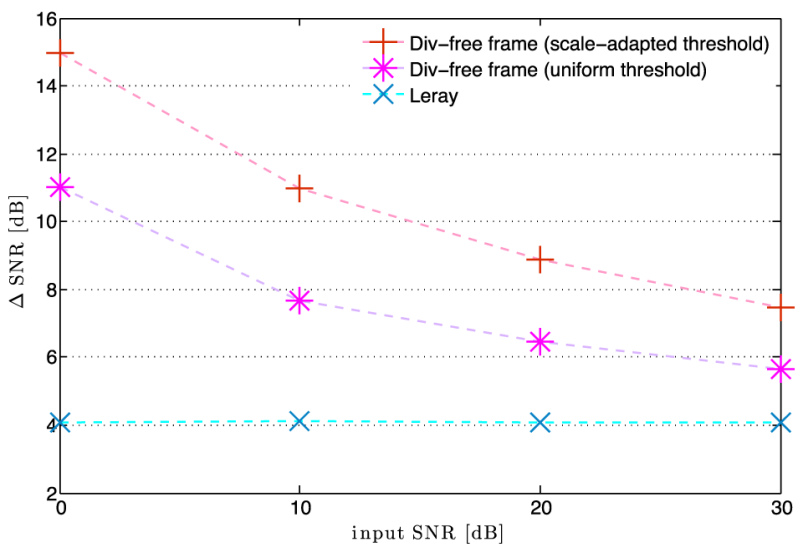

Fig. 2. Denoising results for circle: See text for details.

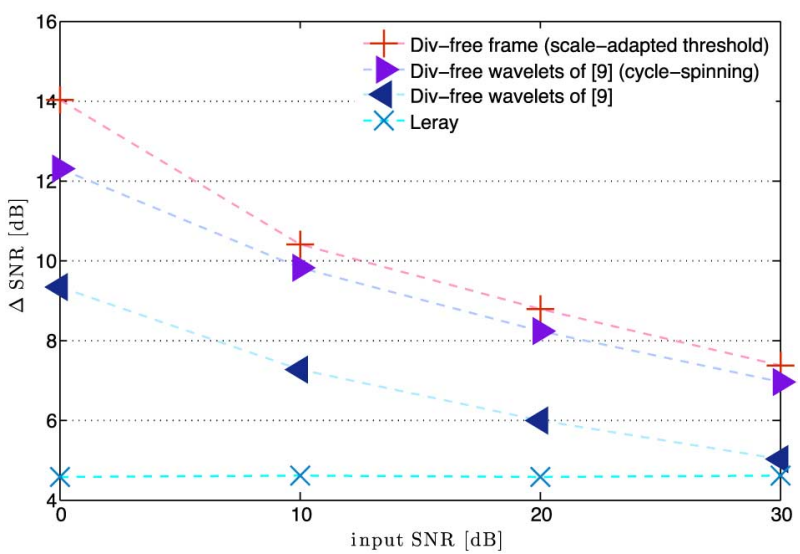

Fig. 3. Denoising results for tube\&torus phantom: See text for details.

First, we investigate the effect of suitably modifying the threshold value for multi-scale decompositions. We use our the divergence-free frame $\mathcal{P} \boldsymbol{\psi}_{j, \boldsymbol{k}, n}$ with 3-scale decomposition for denoising the circle phantom. To make the thresholding scale-adapted, we compute appropriate multiplicative factors by expanding a white Gaussian noise (with a known variance) and measuring the average signal power at each scale. We compare this strategy to simply applying the same threshold value to all scales. By looking at Fig. 2, we see that the scale-adapted thresholding significantly improves the denoising performance of our method.

In the second part of our experiments, we compare our method against the bi-orthogonal divergence-free wavelets of 


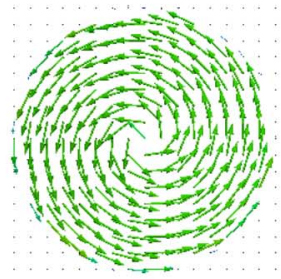

(a)

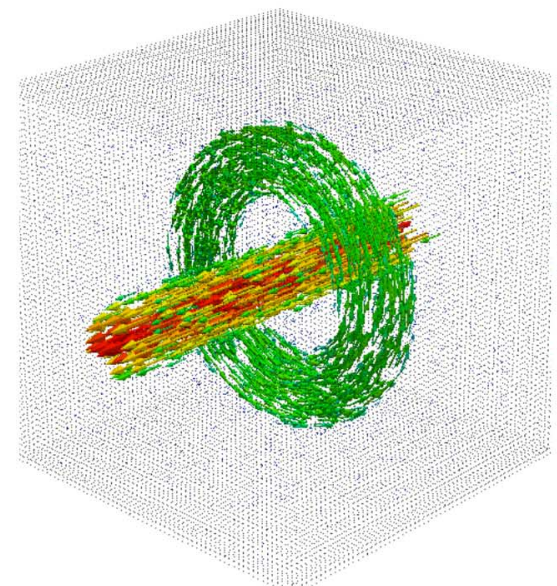

(d)

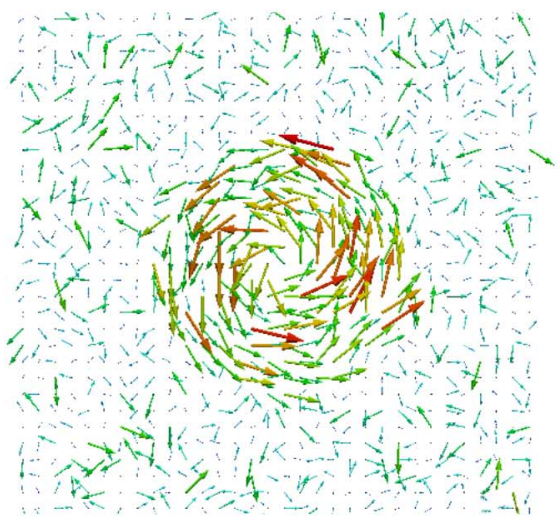

(b)

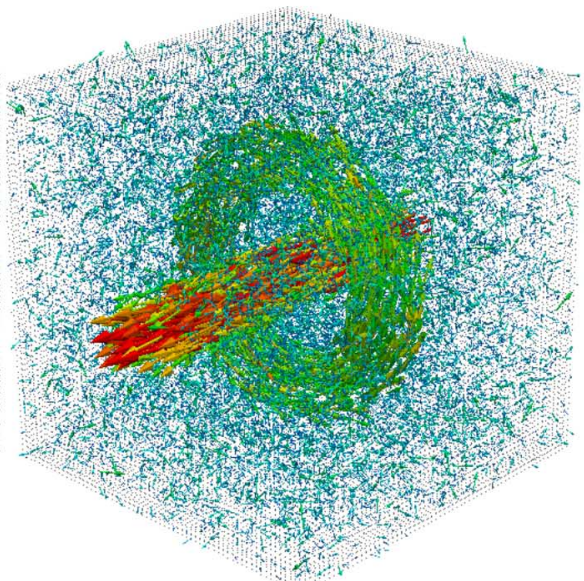

(e)

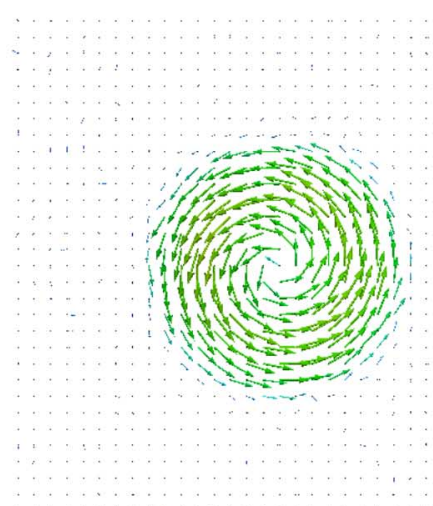

(c)

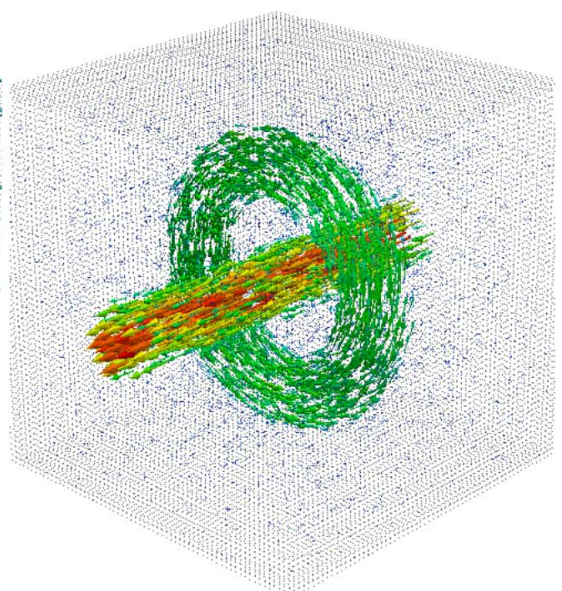

(f)

Fig. 4. Illustration of the denoising results. Original circle and tube\&torus phantoms are shown in (a) and (d), respectively. Noisy vector fields (provided in (b) and (e), and their SNR value is $0 \mathrm{~dB}$ ) are reconstructed by using our divergence-free wavelet frame. The SNR of the reconstructed fields are 14.94, and $14.08 \mathrm{~dB}$ for (c) and (f), respectively.

[9] $]^{1}$. Since our method is redundant (redundancy factor 3), we also include the cycle-spun version of the bi-orthogonal divergence-free wavelets (redundancy factor 8). As a baseline method, Leray projection, which is implemented as a Fourier domain filtering using (6), is incorporated in the comparisons.

The denoising is done as follows. As a pre-processing step, we apply a low-pass filter to the initial noisy data. Recall that our phantoms are band-limited, and the pre-filtering is used for all of the algorithms in question to make the comparison fair. For wavelet methods, 3-scale signal decomposition is performed, scale-adapted thresholding is used, and the threshold values are optimized for the best-possible SNR performance using an oracle.

By inspecting the results given in Fig. 3, we see that the proposed divergence-free frame outperforms the other methods for the simulated field considered in the experiment. Since the construction given in [9] is bi-orthogonal, the wavelet transform does not preserve energy. In other words, the minimization of the $\ell_{2}$-normed error in the wavelet domain does not imply the minimization of the $\ell_{2}$-normed error in the signal domain. The noise statistics also change in the transform domain.

${ }^{1}$ The software is available at http://www.eecs.berkeley.edu/mlustig/Software.html.
However, since our reconstruction is tight, the energy is preserved, and thus the said drawback of bi-orthogonal methods is not observed. This shows the effectiveness of our approach for denoising incompressible vector fields (see Fig. 4 for visual inspection).

\section{CONCLUSION}

We proposed a construction of divergence-free wavelet frames. We based our approach on the observation that applying the Leray projector $\mathcal{P}$ to a standard frame produces a divergence-free version. Furthermore, we used the singular-integral interpretation of $\mathcal{P}$ to show that the derived frame maintains the desirable attributes (decay rate and order of vanishing moments) of the original wavelets.

Based on a particular collection of wavelet frames of $L_{2}\left(\mathbb{R}^{d}\right)$, we proposed divergence-free frames of $\mathcal{H}\left(\mathbb{R}^{d}\right)$. The effectiveness of the two- and three-dimensional variants was illustrated on generated band-limited phantoms. These experiments hint at the potential of our wavelets for denoising impressible flow data, which is modeled as divergence-free.

\section{ACKNOWLEDGMENT}

The authors thank Florence Gavin for her initial contributions to the experimental validation of their construction. 


\section{REFERENCES}

[1] X. Zhou and Y. He, "Using divergence free wavelets for the numerical solution of the 2-D stationary Navier-Stokes equations," Appl. Math. Comput., vol. 163, no. 2, pp. 593-607, 2005.

[2] K. Urban, Wavelets in Numerical Simulation: Problem Adapted Construction and Applications. Berlin, Germany: Springer, 2002, vol. 22.

[3] M. Markl, A. Frydrychowicz, S. Kozerke, M. Hope, and O. Wieben, "4D flow MRI," J. Magn. Reson. Imag., vol. 36, no. 5, pp. 1015-1036, Nov. 2012.

[4] E. Bostan, O. Vardoulis, D. Piccini, P. D. Tafti, N. Stergiopulos, and M. Unser, "Spatio-temporal regularization of flow-fields," in Proc. IEEE Int. Symp. Biomed. Imaging, Apr. 7-11, 2013, pp. 836-839.

[5] F. Ong, M. Uecker, U. Tariq, A. Hsiao, M. T. Alley, S. S. Vasanawala, and M. Lustig, "Robust 4D flow denoising using divergence-free wavelet transform," Magn. Reson. Med., 2014, to be published.

[6] D. Schiavazzi, F. Coletti, G. Iaccarino, and J. K. Eaton, "A matching pursuit approach to solenoidal filtering of three-dimensional velocity measurements," J. Comput. Phys., vol. 263, pp. 206-221, 2014.

[7] G. Battle and P. Federbush, "Divergence-free vector wavelets," Michigan Math. J., vol. 40, no. 1, pp. 181-195, 1993.

[8] P. G. Lemarie-Rieusset, "Analyses multi-résolutions non orthogonales, commutation entre projecteurs et dérivation et ondelettes vecteurs á divergence nulle," Rev. Mat. Iberoamer., vol. 8, no. 2, pp. 221-237, 1992.

[9] E. Deriaz and V. Perrier, "Divergence-free and curl-free wavelets in two dimensions and three dimensions: Application to turbulent flows," J. Turbulence, vol. 7, no. 3, pp. 1-37, 2006.
[10] R. Stevenson, "Divergence-free wavelet bases on the hypercube: Freeslip boundary conditions, and applications for solving the instationary Stokes equations," Math. Comput., vol. 80, no. 275, pp. 1499-1523, 2011.

[11] S. Kadri-Harouna and V. Perrier, "Effective construction of divergence-free wavelets on the square," J. Comput. Appl. Math., vol. 240, pp. 74-86, 2013.

[12] K. Urban, "On divergence-free wavelets," Adv. Comput. Math., vol. 4, no. 1, pp. 51-81, 1995.

[13] A. J. Majda and A. L. Bertozzi, Vorticity and Incompressible Flow. Cambridge, U.K.: Cambridge Univ. Press, 2002, vol. 27.

[14] O. Christensen, An Introduction to Frames and Riesz Bases. Boston, MA, USA: Birkhäuser, 2003.

[15] J.P. Ward, K. N. Chaudhury, and M. Unser, "Decay properties of Riesz transforms and steerable wavelets," SIAM J. Imag. Sci., vol. 6, no. 2, pp. 984-998, 2013.

[16] J. P. Ward and M. Unser, "Harmonic singular integrals and steerable wavelets in $L_{2}\left(\mathbb{R}^{d}\right)$," Appl. Comput. Harmon. Anal., vol. 36, no. 2, pp. 183-197, 2014.

[17] S. Held, M. Storath, P. Massopust, and B. Forster, "Steerable wavelet frames based on the Riesz transform," IEEE Trans. Image Process., vol. 19, no. 3, pp. 653-667, 2010.

[18] M. Unser, N. Chenouard, and D. V. D. Ville, "Steerable pyramids and tight wavelet frames in $L_{2}\left(\mathbb{R}^{d}\right)$," IEEE Trans. Image Process., vol. 20, no. 10 , p. 2705 , Oct. 2011 .

[19] D. L. Donoho and I. M. Johnstone, "Ideal spatial adaptation by wavelet shrinkage," Biometrika, vol. 81, no. 3, pp. 425-455, 1994.

[20] J. Ahrens, B. Geveci, and C. Law, "Paraview: An end-user tool for largedata visualization," The Visualization Handbook, pp. 717-732, 2005. 